\title{
BOUNDED SOLUTIONS OF NONLINEAR DIFFERENCE EQUATIONS
}

\author{
SUI SUN CHENG AND HORNG JAAN LI
}

1. In this note we are concerned with the question of boundedness of solutions of the following difference equation

$$
\triangle\left(p_{k-1} \Delta x_{k-1}\right)=q_{k} f\left(x_{k}\right), \quad k=1,2,3, \ldots
$$

where $p_{k}>0$ for $k=0,1,2, \ldots, f$ is a real function definded on $R$ such that sign $f(x)=\operatorname{sign} x$ and $q_{k} \geq 0$ for $k=1,2,3, \ldots$ In several recent papers $[1,2,3,4]$, oscillatory, periodic and asymptotic behavior of solutions of second order difference equations have been investigated. In particular, the case when $f(x)=x$ and the case $f(x)=x^{r}$ where $r$ is a quotient of odd positive integers, have been studied $[1,3]$.

A solution of (1) is a real sequence $x=\left\{x_{k}\right\}_{0}^{\infty}$ satisfying (1). It is clear that the standard existence and uniqueness theorem holds for (1). It is also not difficult to see that a nontrivial solution $x$ of (1) is eventually nonnegative or eventually nonpositive. Indeed, suppose without loss of generality that $x_{0} \leq 0$. If $x_{k} \leq 0$ for all $k=1,2, \ldots$, our assertion holds. Otherwise, $x_{1} \leq 0, \ldots, x_{N-1} \leq 0$ but $x_{N}>0$ for some $N>0$. Since $x_{N-1}$ and $x_{N}$ cannot be zero simultaneously, we have from (1) that

$$
p_{N} \Delta x_{N}=p_{N-1} \Delta x_{N-1}+q_{N} f\left(x_{N}\right)>0,
$$

which implies that $x_{N+1}>x_{N} \geq 0$, and by induction that $x_{k}>x_{N} \geq 0$ for all $k>N$.

Theorem 1. A nontrivial solution $x$ of $(1)$ is either eventually positive increasing, eventually positive nonincreasing, eventually negative decreasing or eventually negative nondecreasing.

Indeed, if $x_{k} \geq 0$ for $k \geq N$, then

$$
\triangle\left(x_{k} p_{k-1} \triangle x_{k-1}\right)=p_{k}\left(\triangle x_{k}\right)^{2}+q_{k} x_{k} f\left(x_{k}\right) \geq 0, \quad k>N .
$$

If $\triangle x_{k} \leq 0$ for all $k \geq N$, then $x$ is nonincreasing for $k \geq N$. Otherwise there is some $m \geq N$ such that $\Delta x_{m}>0$. In this case, $x_{m+1}>x_{m} \geq 0$ which implies $x_{m+1} p_{m} \Delta x_{m}>$

Received March 7, 1989; revised June 3, 1989. 
0 . But then $\Delta\left(x_{m+1} p_{m} \Delta x_{m}\right) \geq 0$ implies $x_{m+2} \Delta x_{m+1}>0$. Since $x_{m+2} \geq 0$, we have $\Delta x_{m+1}>0$. By similar arguments, we show by induction that $\Delta x_{k}>0$ for $k>m$. It is clear that if $x$ is an eventually nonnegative nonincreasing then it is an eventually positive nonincreasing. The case that $x_{k}<0$ for $k \geq N$ is similarly proved.

2. It is not clear whether (1) has a bounded solution. However, if $f$ is continuous, then it can be shown that (1) has a positive nonincreasing solution so that a bounded solution exists. The proof of this statement is similar to that of Theorem 3.2 in [3] and is thus omitted. It is also not known what conditions are sufficient or necessary for the boundedness of all solutions of (1). Some of these conditions will be given below.

Theorem 2. Suppose $f$ is nondecreasing, $f(x) / x$ is nonincreasing for $x>0, q_{k} \neq 0$ for infinitely many $k$, and

$$
\sum_{n=1}^{\infty} \sum_{k=1}^{n} \frac{q_{k}}{p_{n}}<\infty
$$

then every eventually positive solution of (1) is bounded.

Proof. Suppose $x$ is an eventually positive and unbounded solution of (1). By Theorem 1, we may assume without loss of generality that $x_{k}>0$ and $\Delta x_{k}>0$ for $k \geq N$. From (1), we have

$$
\begin{aligned}
q_{k} & =\frac{\Delta\left(p_{k-1} \Delta x_{k-1}\right)}{f\left(x_{k}\right)}=\frac{p_{k} \Delta x_{k}}{f\left(x_{k}\right)}-\frac{p_{k-1} \Delta x_{k-1}}{f\left(x_{k}\right)} \\
& \geq \frac{p_{k} \Delta x_{k}}{f\left(x_{k}\right)}-\frac{p_{k-1} \Delta x_{k-1}}{f\left(x_{k-1}\right)}=\Delta\left\{\frac{p_{k-1} \Delta x_{k-1}}{f\left(x_{k-1}\right)}\right\}, \quad k>N .
\end{aligned}
$$

Summing both sides of (3) from $k=N+1$ to $k=m$, we obtain

$$
\sum_{k=N+1}^{m} q_{k}+\frac{p_{N} \Delta x_{N}}{f\left(x_{N}\right)} \geq \frac{p_{m} \Delta x_{m}}{f\left(x_{m}\right)}
$$

Since $f(x) / x$ is nonincreasing for $x>0$, we have from (4) that

$$
\frac{\Delta x_{m}}{x_{m}} \leq \frac{f\left(x_{N}\right) \Delta x_{m}}{x_{N} f\left(x_{m}\right)} \leq \frac{f\left(x_{N}\right)}{x_{N}} \sum_{k=N+1}^{m} \frac{q_{k}}{p_{m}}+\frac{p_{N} \Delta x_{N}}{p_{m} x_{N}} .
$$

Let $g(t)=x_{m}+(t-m) \Delta x_{m}$ for $m \leq t \leq m+1$. Then $g^{\prime}(t)=\Delta x_{m}$ and $g(t) \geq x_{m}$ for $m \leq t \leq m+1$. Hence

$$
\frac{\Delta x_{m}}{x_{m}}=\int_{m}^{m+1} \frac{g^{\prime}(t)}{x_{m}} d t \geq \int_{m}^{m+1} \frac{g^{\prime}(t)}{g(t)} d t=\log \left(x_{m+1}\right)-\log \left(x_{m}\right) .
$$


If we now sum $\Delta x_{m} / x_{m}$ from $m=N+1$ to $m=n$, then (5) and (6) imply

$$
\begin{aligned}
\log \left(x_{n+1}\right)-\log \left(x_{N+1}\right) & \leq \sum_{m=N+1}^{n} \frac{\triangle x_{m}}{x_{m}} \\
& \leq \frac{f\left(x_{N}\right)}{x_{N}} \sum_{m=N+1}^{n} \sum_{k=N+1}^{m} \frac{q_{k}}{p_{m}}+\frac{p_{N} \Delta x_{N}}{x_{N}} \sum_{m=N+1}^{n} \frac{1}{p_{m}} .
\end{aligned}
$$

Next choose $T \geq N+1$ such that $q_{T} \neq 0$, then by (2)

$$
q_{T} \sum_{n=T}^{\infty} \frac{1}{p_{n}} \leq \sum_{n=1}^{\infty} \frac{1}{p_{n}} \sum_{k=1}^{\infty} q_{k}<\infty
$$

so that $\left\{\log \left(x_{n}\right)\right\}$ is bounded. This contradiction completes our proof.

We remark that if the assumption, that $f(x) / x$ is nonincreasing for $x>0$, is replaced by the assumption that $f(x) / x$ is nondecreasing for $x<0$, then we may conclude that every eventually negative solution of $(1)$ is bounded. Analogous remarks also hold for the following theorem.

Theorem 3. Suppose $f(x) / x$ is nonincreasing for $x>0, q_{k} \neq 0$ for infinitely many $k$ and that

$$
\sum_{n=1}^{\infty} q_{n} \sum_{k=1}^{\infty} \frac{1}{p_{k}}<\infty
$$

then every eventually positive solution of (1) is bounded.

Proof. Assume that $x$ is an eventually positive solution of (1). We first show that the sequence $\left\{p_{k} \Delta x_{k}\right\}$ is bounded. Indeed, if $x$ is eventually nonincreasing, then the sequence $\left\{p_{k} \Delta x_{k}\right\}$ is eventually nonpositive and nondecreasing since $\Delta\left(p_{k} \Delta x_{k}\right)=$ $q_{k+1} f\left(x_{k+1}\right) \geq 0$ for all large $k$. If $x$ is eventually increasing such that $x_{k}>0$ and $\Delta x_{k}>0$ for $k \geq N$. Then

$$
\Delta\left\{\frac{x_{k+1}}{p_{k} \Delta x_{k}}\right\}=\frac{p_{k} \Delta x_{k} \Delta x_{k+1}-q_{k+1} x_{k+1} f\left(x_{k+1}\right)}{p_{k} p_{k+1} \Delta x_{k} \Delta x_{k+1}} \leq \frac{1}{p_{k+1}}
$$

for $k \geq N$. Summing both sides from $k=N$ to $k=m-1$, we have

$$
\frac{x_{m+1}}{p_{m} \Delta x_{m}} \leq \frac{x_{N+1}}{p_{N} \Delta x_{N}}+\sum_{k=N+1}^{m} \frac{1}{p_{k}} \text {. }
$$

Since $f(x) / x$ is nonincreasing for $x \neq 0$, we have from (1) and the above inequality that

$$
\begin{aligned}
\frac{\Delta\left(p_{m} \Delta x_{m}\right)}{p_{m} \Delta x_{m}} & =\frac{q_{m+1}}{p_{m} \Delta x_{m}} f\left(x_{m+1}\right) \\
& \leq \frac{q_{m+1}}{p_{m} \Delta x_{m}} \frac{f\left(x_{N+1}\right) x_{m+1}}{x_{N+1}} \\
& \leq \frac{q_{m+1} f\left(x_{N+1}\right)}{p_{N} \Delta x_{N}}+\frac{q_{m+1} f\left(x_{N+1}\right)}{x_{N+1}} \sum_{k=N+1}^{m} \frac{1}{p_{k}} .
\end{aligned}
$$


As in the proof of Theorem 2, we then obtain

$$
\begin{gathered}
\log \left(p_{n+1} \Delta x_{n+1}\right)-\log \left(p_{N+1} \Delta x_{N+1}\right) \leq \sum_{m=N+1}^{n} \frac{\Delta\left(p_{m} \Delta x_{m}\right)}{p_{m} \Delta x_{m}} \\
\leq \frac{f\left(x_{N+1}\right)}{p_{N} \Delta x_{N}} \sum_{m=N+1}^{n} q_{m+1}+\frac{f\left(x_{N+1}\right)}{x_{N+1}} \sum_{m=N+1}^{n} \sum_{k=N+1}^{m} \frac{q_{m+1}}{p_{k}} .
\end{gathered}
$$

Since (7) implies that

$$
\sum_{m=1}^{\infty} q_{m}<\infty
$$

thus $\left\{\log \left(p_{k} \Delta x_{k}\right)\right\}$ and $\left\{p_{k} \Delta x_{k}\right\}$ are bounded.

Now that

$$
\triangle x_{k} \leq \frac{M}{p_{k}}, \quad k \geq N
$$

where $M$ is an upper bound for the sequence $\left\{p_{k} \Delta x_{k}\right\}$, we have by summing from $k=N+1$ to $k=n$ that

$$
x_{n+1} \leq x_{N+1}+\sum_{k=N+1}^{n} \frac{M}{p_{k}} .
$$

Again since (7) implies

$$
\sum_{k=N+1}^{\infty} \frac{1}{p_{k}}<\infty
$$

the solution $x$ must be bounded. Q.E.D.

Theorem 4. Suppose $f(x)$ is nonincreasing for $x>0, q_{k} \neq 0$ for infinitely many $k$ and (2) holds. Then every eventually positive solution of (1) is bounded.

Proof. Assume that $x$ is an eventually positive solution of $(1)$ and that $x_{k}>0$ and $\Delta x_{k}>0$ for $k \geq N$. Since $f\left(x_{k+1}\right) \leq f\left(x_{k}\right)$ for $k \geq N$, we have

$$
\Delta\left(p_{k-1} \Delta x_{k-1}\right)=q_{k} f\left(x_{k}\right) \leq q_{k} f\left(x_{N}\right), \quad k \geq N
$$

so that

$$
\Delta x_{n} \leq p_{n}^{-1} p_{N} \Delta x_{N}+f\left(x_{N}\right) p_{n}^{-1} \sum_{k=N+1}^{n} q_{k}, \quad n>N
$$

and

$$
x_{m+1} \leq x_{N+1}+p_{N} \Delta x_{N} \sum_{n=N+1}^{m} p_{n}^{-1}+f\left(x_{N}\right) \sum_{n=N+1}^{m} p_{n}^{-1} \sum_{k=N+1}^{n} q_{k} .
$$

Since (2) implies

$$
\sum_{n=N+1}^{m} p_{k}^{-1}<\infty
$$

thus $x$ is bounded. 
We remark that if the assumption, that $f(x)$ is nonincreasing for $x>0$, is replaced by the assumption that $f(x)$ is nondecreasing for $x<0$, then we may conclude that every eventually negative solution of (1) is bounded.

3. We now turn our attention to unbounded solutions. Note first that, in view of Theorem 1, an unbounded solution is either an eventually positive increasing or negative decreasing solution.

Theorem 5. Suppose $f$ is nondecreasing, $q_{k} \neq 0$ for infinitely many $k$, and

$$
\sum_{n=1}^{\infty} \sum_{k=1}^{\infty} \frac{q_{k}}{p_{n}}=\infty
$$

then every eventually positive increasing or negative decreasing solution $x$ of (1) is unbounded.

Proof. Suppose $x_{k}>0$ and $\Delta x_{k}>0$ for $k \geq N$. Since

$$
\triangle\left(p_{k-1} \Delta x_{k-1}\right)=q_{k} f\left(x_{k}\right) \geq q_{k} f\left(x_{N}\right), \quad k \geq N
$$

by summing both sides from $k=N+1$ to $k=n$, we obtain

$$
\triangle x_{n} \geq p_{n}^{-1} p_{N} \Delta x_{N}+p_{n}^{-1} f\left(x_{N}\right) \sum_{k=N+1}^{n} q_{k}, \quad n \geq N+1 .
$$

Again, by summing both sides of (10) from $n=N+1$ to $n=m$, we obtain

$$
x_{m+1} \geq x_{N}+p_{N} \Delta x_{N} \sum_{n=N+1}^{m} p_{n}^{-1}+f\left(x_{N}\right) \sum_{n=N+1}^{m} p_{n}^{-1} \sum_{k=N+1}^{n} q_{k},
$$

which, in view of (8), implies $x_{m} \rightarrow \infty$ as $m \rightarrow \infty$. Q.E.D.

The assumption that $q_{k} \neq 0$ for infinitely many $k$ can be removed if a stronger condition than (8) is assumed.

Theorem 6. Suppose

$$
\sum_{k=1}^{\infty} \frac{1}{p_{k}}=\infty
$$

then every eventually positive increasing or negative decreasing solution $x$ of (1) is unbounded. 
Proof. Suppose $x_{k}>0$ and $\Delta x_{k}>0$ for $k \geq N$, then similar to (11), we may derive the inequality

$$
x_{m+1} \geq x_{N}+p_{N} \Delta x_{N} \sum_{n=N}^{m} p_{n}^{-1} .
$$

our assertion now follows from (12).

When $f(x)=x$ Theorems 2 and 5 imply the following consequence which has been derived previously $[1$, Theorem 4].

Corollary. Suppose $f(x)=x$ and $q_{k} \neq 0$ for infinitely many $k$, then every solution of (1) is bounded if and only if (2) holds.

The above Corollary, since it contains a necessary and sufficient condition, indicates that Theorems 2 and 6 are sharp.

\section{References}

[1] S. S. Cheng, H. J. Li and W. T. Patula, "Bounded and zero convergent solutions of second order difference equations," J. Math. Anal. Appl., 141(1989), 463-483.

[2] A. Drozdowicz and J. Popenda, "Asymptotic behavior of the solutions of the second order difference equation," Proc. AMS, 99(1987), 135-140.

[3] J. W. Hooker and W. T. Patula, "A second order nonlinear difference equation: oscillation and asymptotic behavior," J. Math. Anal. Appl., 91(1983), 9-29.

[4] F. Weil, "Existence theorem for the difference equation $y_{n+1}-2 y_{n}+y_{n-1}=h^{2} f\left(y_{n}\right)$," Internat. J. Math. Sci., 3(1)(1980), 69-77. 